\title{
La voz de los estudiantes ante la reconfiguración de la actividad docente universitaria en la pandemia
}

\author{
Hannia Gonzalez-Urango ${ }^{a^{*}}$; Carmen Corona-Sobrino ${ }^{\text {ab }}$; Ivan Ligardo-Herrera ${ }^{\mathfrak{c},}$
}

angenio (CSIC-UPV), Universitat Politècnica de València, 46022 Valencia, Spain, bUniversidad de Alicante, Departamento de Sociología I y 'Instituto Ingeniería Energética IU-IIE, Universitat Politècnica de València, 46022 Valencia, Spain.*Correspondencia: hkgonzal@upv.es

\section{Abstract}

The global pandemic caused by the emergence of the new COVID-19 has led to changes in all spheres of life as we knew it. Education has not been exempt. Institutions of the entire planet closed their rooms and the students turned on their computers to access an unusual teaching format in Spanish education. In the case of Higher Education in Spain, distance education has continued to be present. In this communication, we give a voice to the students of two public universities in the Valencian Community in order to know first-hand how they have been affected by this change. Through a questionnaire $(n=86)$ we set out to find out how this process has been by studying four dimensions: quality and satisfaction with teaching, connectivity and infrastructure, environment and personal problems. The students have considered an important change in the quality of the teaching received compared to the period before the crisis, as well as a lack of concentration and motivation to continue this type of teaching. Finally, we consider a series of lessons learned in order to continue living, for the moment, with this health emergency.

Keywords: students, distance education, virtual education, quality, satisfaction, connectivity, infrastructure, study environment, personal problems

\section{Resumen}

La pandemia global provocada por la irrupción del nuevo COVID-19 ha supuesto cambios en todas las esferas de la vida tal y como la conocíamos. La educación no se ha quedado exenta. Los centros educativos de todo el planeta cerraron sus puertas y el alumnado encendió sus ordenadores para acceder a un formato de docencia hasta ahora poco habitual en la enseñanza española. En el caso de la Educación Superior en España, la educación a distancia ha seguido presente. En esta comunicación, damos voz al alumnado de dos universidades públicas de la Comunidad Valenciana con el fin de conocer de primera mano cómo se han visto afectados por este cambio. A través de un cuestionario (n=86) nos planteamos conocer cómo ha sido este proceso estudiando cuatro dimensiones: calidad y satisfacción con la docencia recibida, conectividad e infraestructura, entorno y ambiente de estudio, y problemas personales. El alumnado ha manifestado un importante cambio en la calidad de la docencia recibida respecto al periodo anterior a la crisis, así como una elevada falta de concentración y motivación para seguir este tipo de docencia. Por último, nos planteamos una serie de lecciones aprendidas de cara a seguir conviviendo, por el momento, con esta emergencia sanitaria.

Palabras clave: estudiantes, alumnado, educación a distancia, educación virtual, calidad, satisfacción, conectividad, infraestructura, ambiente de estudio, problemas personales 


\section{Introducción}

La crisis sanitaria provocada por la COVID 19 y la consiguiente declaración de pandemia mundial, ha supuesto un desafío para la educación a todos los niveles. Esta crisis sanitaria ha incitado cambios en las maneras de comprender e interpretar todas las esferas de la sociedad, tal y como la conocíamos. En este sentido, la universidad ha requerido de una adaptación de sus procesos y de una reconfiguración de sus servicios. Las tres misiones de la universidad, docencia, investigación y transferencia (Molas \& CastroMartínez, 2007), están en un proceso de adaptación a la nueva normalidad.

El caso que aquí nos compete, la docencia, está en un proceso continuo de reconfiguración, ha pasado del espacio físico a la virtualidad del aula, se han creado nuevas plataformas de intercambio y se han incrementado los canales de comunicación a una velocidad inaudita $(\mathrm{Mu}, 2020)$. Según la $\mathrm{UNESCO}^{1}$ a 1 de abril de 2020, 173 países habían cerrado sus centros escuelas y universidades afectando a 1.600 millones de estudiantes. El 84,3\% del alumnado total matriculado en instituciones educativas a nivel mundial se vio afectado por estas medidas. Esta situación sobrevenida supuso una reinterpretación de la experiencia educativa tradicional: de la presencialidad en el aula pasamos a la virtualidad desde el hogar.

En este sentido las universidades han tenido que experimentar un proceso forzoso de transformación al modelo online, pero sin pretender asumir este modelo integralmente. Es decir, se ha creado un modelo híbrido para solventar una situación, a priori temporal, pero que dura actualmente más de un año. En el tránsito de un modelo a otro hay ciertas competencias y características que es imposible asumir (Area Moreira, Bethencourt Aguilar, \& Martín Gómez, 2020). Hay quien afirma que "la situación actual no es enseñanza en línea sino más bien Educación a distancia de emergencia" ${ }^{2}$. Se ha producido un auge de herramientas de e-learning como Microsoft Teams o Zoom y se han tratado de adaptar las formas de evaluación a esta situación (Ismaili, 2021). Sin embargo, la inmediatez de la situación y la dificultad del tránsito repentinamente de un modelo a otro ha hecho que el pilar de la educación superior se haya visto comprometido. Por ejemplo, la evaluación ha sido una de las principales quejas debido a la incapacidad actual para detectar actos fraudulentos durante la realización de las pruebas. Por ello, las recomendaciones y medios que permiten minimizar su impacto han sido de especial relevancia (González, Marco, \& Medina, 2020).

Son muchos los frentes abiertos y las líneas de estudio que ha deparado y depara esta disruptiva social. En esta comunicación queremos centrarnos en la educación superior en el contexto de dos universidades de la Comunidad Valenciana y ofrecer una visión de la problemática desde el alumnado. La novedad del trabajo reside en darle voz a los estudiantes con el fin de comprender cómo se han visto afectados por la pandemia y cómo están haciendo frente a los cambios. Asimismo, en línea con los Objetivos de Desarrollo del Milenio y la importancia de incorporar la perspectiva de género en las investigaciones, los datos estarán recogidos y analizados teniendo en cuenta esta consideración. De hecho, parte del interés del estudio reside en las dos áreas de conocimiento estudiadas: Ciencias Sociales e Ingeniería, completamente distintas en sus modos de trabajo y concepciones. Estas titulaciones se imparten al alumnado $(\mathrm{n}=86)$ de dos universidades de la Comunidad Valenciana (Universidad de Alicante y Universidad Politécnica de Valencia) en dos cursos diferentes.

\footnotetext{
${ }^{1}$ Ver gráfico de la evolución mundial del cierre de instituciones educativas y porcentaje de alumnado afectado por las restricciones de la pandemia en https://es.unesco.org/covid19/educationresponse Consultado a 21 de marzo de 2021.

${ }^{2}$ Artículo en la revista Magisterio https://www.magisnet.com/2020/05/la-educacion-que-viene-un-modelo-hibrido-y-mas-tecnologico/ publicado el 12 de mayo 2020.
} 
Durante décadas se ha constatado el sesgo de género, a favor de los hombres, en la elección de carreras STEM (por sus siglas en inglés Ciencia, Tecnología, Ingeniería y Matemáticas) frente a una mayor feminización de los estudios sociales. Los resultados muestran que, en la mayoría de los países, las alumnas son iguales o mejores que los alumnos en ciencias y además están perfectamente capacitadas para elegir estudios STEM en bachillerato y en la universidad. Sin embargo, cuanto más alto aparece el país en los baremos de igualdad, el número de alumnas que eligen una carrera STEM es menor y, por tanto, el sesgo entre géneros aumenta. Los autores lo llaman la "paradoja educacional de la igualdad de género": a más igualdad más distancia, en STEM, entre hombres y mujeres (Stoet \& Geary, 2018). Por ello, consideramos que en una situación de emergencia mundial, como la que estamos viviendo actualmente, también es fundamental tener en cuenta esta perspectiva de género, para que, en caso de ser necesario, se puedan tomar las medidas precisas.

\subsection{El salto a la educación a distancia}

En España conviven dos modelos de educación superior, las universidades presenciales y las universidades online. Según el último informe publicado por el Ministerio de Universidades en 2020 "Datos y Cifras del Sistema Universitario Español" sobre el curso 2019-2020, en España existen 83 universidades oficiales entre públicas y privadas de las cuales 75 son presenciales frente a 6 no presenciales y 2 de régimen especial. De las no presenciales sólo 1 tienen titularidad pública (Universidad Nacional de Educación a Distancia) mientras que las otras 6 son de carácter privado.

Es fundamental comenzar dejando claro que la situación sobrevenida provocada por la COVID-19 no ha implicado en todos los casos un cambio a la educación online si no pasar a la educación a distancia. Teniendo en cuenta la clasificación realizada por Durán-Rodríguez (2015) podemos clasificar los tipos de docencia de la siguiente manera, atendiendo además a sus ventajas y desventajas:

Docencia presencial: se caracteriza por la asistencia regular a encuentros presenciales en espacios físicos preestablecidos y por la clase magistral (Durán-Rodríguez, 2015).

\section{Ventajas}

- Relación estrecha entre los docentes y los compañeros y compañeras de clase

- Permite, con mayor o menor éxito, atender a grupos numerosos

- Permite determinadas estrategias docentes que favorecen el aprendizaje y la retención de contenido

- Es un espacio propicio para que el docente pueda explotar sus competencias

\section{Desventajas}

- Exige la presencia física ya que sin ella no se puede seguir la asignatura/s

- Poca participación

- No favorece un aprendizaje autónomo

- Dificulta otro tipo de conocimiento que no sea la asimilación pasiva de contenidos

Docencia virtual: (Concha Vergara, 2014; Dixon, Dixon, \& Siragusa, 2007) definen el e-learning como el proceso donde los estudiantes interactúan entre ellos, con el profesorado y con los materiales vía internet. Son numerosos los estudios que antes de la pandemia se habían concentrado en analizar los efectos, el rendimiento y las actitudes ante este nuevo modelo educativo (Bertea, 2009; Campbell et al., 2010; Struyven, Dochy, \& Janssens, 2005). Sin embargo, nadie imaginaba una convergencia mundial a este 
respecto orquestada en tiempo record. En este caso el alumnado tiene múltiples opciones de participación de manera sincrónica (videoconferencia, chat interactivo) y asincrónica (foro, correo electrónico).

\section{Ventajas}

- Permite atender a la heterogeneidad de las situaciones de los estudiantes

- Favorece el uso de herramientas que potencian otra serie de competencias menos habituales

- Itinerario flexible de aprendizaje con una variedad de propuestas y tareas más orientadas al trabajo autónomo (Area Moreira et al., 2020)

\section{Desventajas}

- Desincentiva las relaciones sociales

- Desincentiva la participación e incluso incentiva el "anonimato" bajo el respaldo del sistema virtual

- Requiere de habilidades tecnológicas por parte del profesorado

Docencia a distancia también denominada blended learning podemos definirla como "una modalidad educativa donde se entremezclan tiempos y acciones de docencia y aprendizaje desarrolladas tanto en espacios físicos -aulas, seminarios, laboratorios- como a través de entornos virtuales o en línea. Es decir, consiste en desarrollar procesos formativos donde se combinan actividades o tiempos académicos implementados en entornos presenciales con otros tiempos y tareas puestos en práctica a través de espacios digitales" (Area Moreira et al., 2020). Sus potenciales y limitaciones son también de sobra conocidas en la literatura científica (Bartolomé Pina, García Ruiz \& Aguaded Gómez, 2018).

\section{Ventajas}

- Permite la no presencialidad

- Verdadera autonomía para el aprendizaje

- Retroalimentación a título individual lo que favorece el aprendizaje

- Posibilita ofrecer actividades sincrónicas y asincrónicas con sus beneficios y limitaciones (Scull, Phillips, Sharma, \& Garnier, 2020).

\section{Desventajas}

- Requiere de una cuidada planificación de la mediación con el alumnado

- Requiere de canales de comunicación precisos

- Requiere de conocimiento tecnológicos y de formación específica en competencias virtuales

- No permite las relaciones sociales entre el alumnado

\subsection{Principales problemas de la educación a distancia}

La adaptación de la docencia a distancia ha afectado a todos los agentes implicados en el proceso educativo, instituciones, profesorado y alumnado. En esta comunicación queremos centrarnos en los efectos que el paso a una educación no presencial ha tenido para el alumnado. Según la bibliografía consultada, no muy abundante dado que es un fenómeno muy reciente, los principales problemas del alumnado se concentran a nivel mundial en tres dimensiones (Fig. 1) (Ismaili, 2021; Mu, 2020; Scull, Phillips, Sharma, \& Garnier, 2020), trataremos con este estudio de ver cuál es la situación en el caso de las universidades de la Comunidad Valenciana. 


\section{Disponibilidad de recursos}

- Recursos materiales: dispositivos electrónicos y conexión estable a internet

- Recursos del entorno: ubicaciones y espacios físicos que permitan un seguimiento óptimo de la docencia

\section{Competencias digitales}

- Adaptación a las nuevas tecnologías con un propósito educativo

- Adaptación a unas nuevas exigencias y al trabajo de distintas competencias

\section{Cuestiones personales}

- Falta de motivación para el desarrollo continuado de las clases y la denominada "brecha de interés"

- Problemas de índole psicológica

- Problemas de índole económica

Fig. 1 Principales problemas del alumnado durante la pandemia provocada por el covid-19. Elaboración propia según la literatura consultada

\section{Objetivos}

El objetivo principal de este trabajo ha sido dar voz al alumnado de estudios universitarios en las Universidades de Alicante y Universidad Politécnica de Valencia en dos áreas de conocimiento, Ciencias Sociales e Ingeniería, para conocer de primera mano cómo se han visto afectados por la pandemia mundial y la consecuente reconfiguración de la actividad universitaria. Para ello nos planteamos la siguiente pregunta de investigación: ¿Cómo perciben el alumnado matriculado en estudios de educación superior en España los efectos de la pandemia sobre la docencia recibida y su rendimiento?

En este sentido nos planteamos una serie de objetivos secundarios:

O1. Explorar las distintas percepciones del alumnado sobre los efectos que ha tenido la pandemia en cuatro niveles de acción: calidad de la docencia recibida, problemas de conectividad e infraestructura para el desarrollo de la docencia online, ambiente de estudio y problemas personales surgidos a raíz de la situación de crisis sanitaria.

O2. Conocer si existen diferencias de género significativas en los efectos de la pandemia sobre el alumnado universitario.

O3. Reflexionar y discutir algunas lecciones aprendidas sobre la educación en la pandemia que sirvan para la mejora de este proceso en un horizonte temporal más amplio.

\section{Desarrollo de la Innovación: Propuesta metodológica}

Este estudio se propone conocer los desafíos de la educación a distancia en el contexto de la pandemia para los estudiantes. Aquí precisamente es donde reside la innovación del estudio: recoger las percepciones del alumnado, como eje central de la educación. La contribución principal que hace este trabajo es identificar 
los desafíos de la educación no presencial, desde diferentes dimensiones (calidad, entorno, infraestructura, problemas personales, etc.,) en el contexto de la pandemia y desde la perspectiva de los estudiantes.

Para la captura de la información, inicialmente se realizaron entrevistas semiestucturadas con preguntas de satisfacción y preguntas abiertas con el fin de establecer las categorías de análisis. Posteriormente, se diseñó el cuestionario que se distribuyó entre al alumnado de dos universidades de la Comunidad Valenciana (Universidad de Alicante y Universidad Politécnica de Valencia) de las áreas de conocimiento de Ingeniería y Ciencias sociales.

Los cuestionarios fueron aplicados de manera digital empleando la plataforma SurveyMonkey. Se realizó de manera digital para facilitar la recolección de respuestas y que el encuestado no se viera influenciado de ninguna manera por un encuestador, puesto que las preguntas abordan temas de comportamiento y percepción personales. El cuestionario aplicado consta de 24 preguntas, divididas en 9 bloques, con una duración aproximada de 12 minutos. En la Fig. 2 se muestra los pasos seguidos para el desarrollo del presente trabajo.

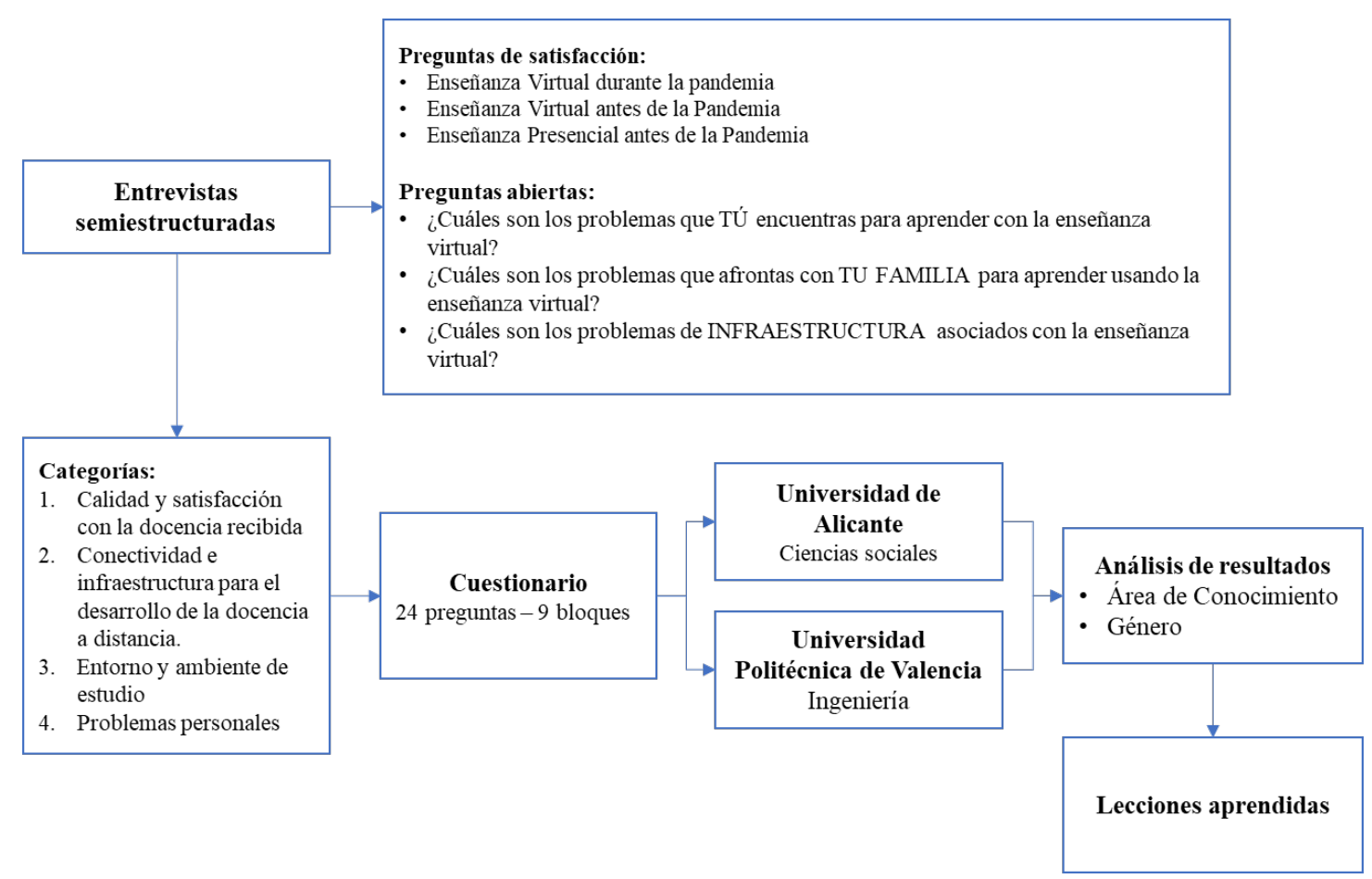

Fig. 2 Diagrama de proceso para el desarrollo de la propuesta.

\section{Resultados}

Los resultados no mostraron grandes diferencias en relación con el género del alumnado, pero si en relación con el área de conocimiento. En este sentido, constatamos la necesidad de tener en consideración la dimensión de género en la investigación según recomienda la normativa europea y estatal (Commission, 2011; European Commission, 2019) aunque luego no existan diferencias significativas en su análisis. Por lo tanto, a continuación, los resultados se presentan resaltando principalmente el área de conocimiento cuando se encontraron diferencias representativas. 


\subsection{Principales características de la población encuestada}

Como se aprecia en la Tabla 1, donde se resume las principales características de la población encuestada, Los participantes son estudiantes de grado principalmente (84\%) en el rango de edad entre 20-24 años. La proporción de participantes fue ligeramente mayor para el área de ingenierías (55\%) en relación con ciencias sociales $(45 \%)$ y equivalente entre mujeres y hombres. Sin embargo, vale la pena resaltar, que es evidente la diferencia entre el número de mujeres y hombres en cada área de conocimiento. Reflejando claramente la diferencia estructural que existe en cada disciplina más o menos feminizada o masculinizada, tal y como se mencionó anteriormente en el informe del Ministerio de Universidades (Datos y Cifras del Sistema Universitario Español, 2020).

Tabla 1. Principales características de la población encuestada

\begin{tabular}{ccccc}
\hline Área de Conocimiento & Femenino & Masculino & NR & Total \\
\hline Ciencias Sociales (UdeA) & 29 & 10 & & 39 \\
Ingenierías (UPV) & 14 & 31 & 2 & 47 \\
\hline Total & $\mathbf{4 3}$ & $\mathbf{4 1}$ & $\mathbf{2}$ & $\mathbf{8 6}$ \\
\hline
\end{tabular}

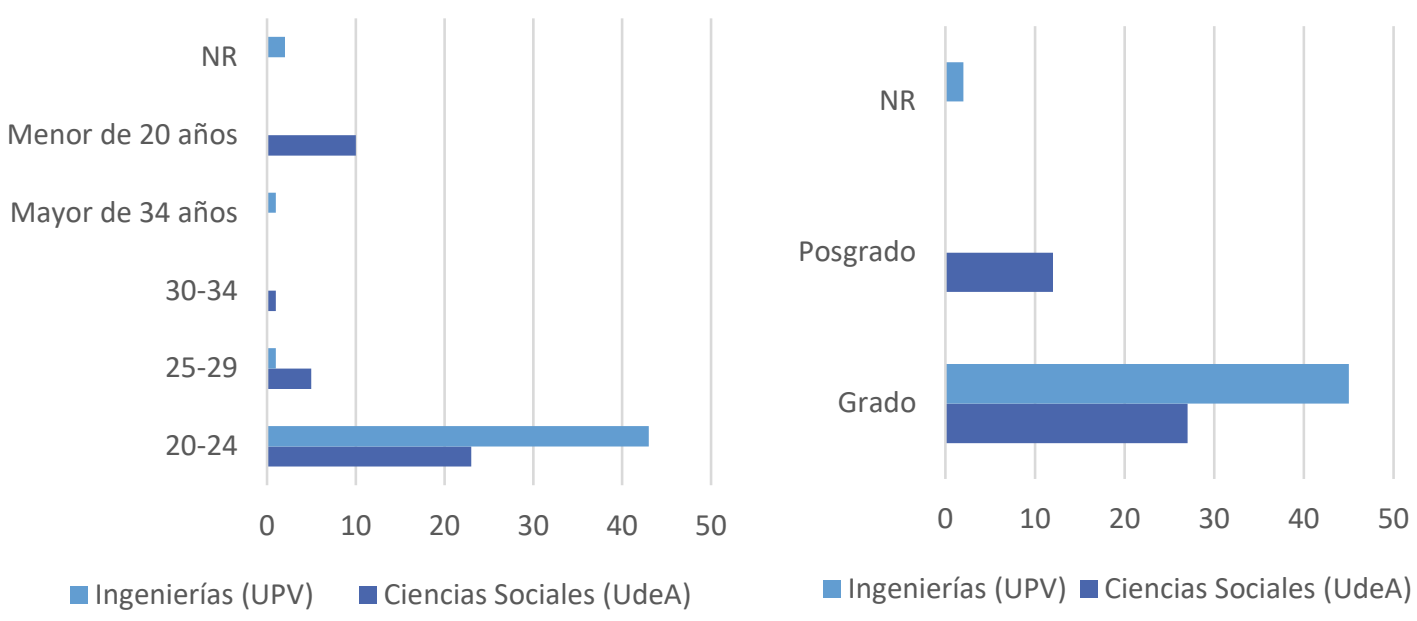

Fig. 3 Características demográficas: Edad y estudios.

Todos los participantes han estado recibiendo clases a distancia como resultado de la emergencia sanitaria provocada por la COVID-19. La mayoría de ellos en modalidad híbrida (parte del curso implica contacto en tiempo real, pero no todo) o virtual síncrona (en tiempo real). Sin embargo, el $73 \%$ nunca habían recibido algún tipo de formación a distancia o virtual. Lo que indica que la situación fue totalmente inédita para la mayoría.

\subsection{Calidad y satisfacción con la docencia recibida}

En términos generales el alumnado estaba más satisfecho con el modelo tradicional de clases antes de la pandemia, en contraste con la situación actual. La satisfacción con las clases a distancia actuales se concentra entre los valores más neutros y bajos.

(c)) BY-NC-ND 2021, Universitat Politècnica de València

Congreso In-Red (2021) 


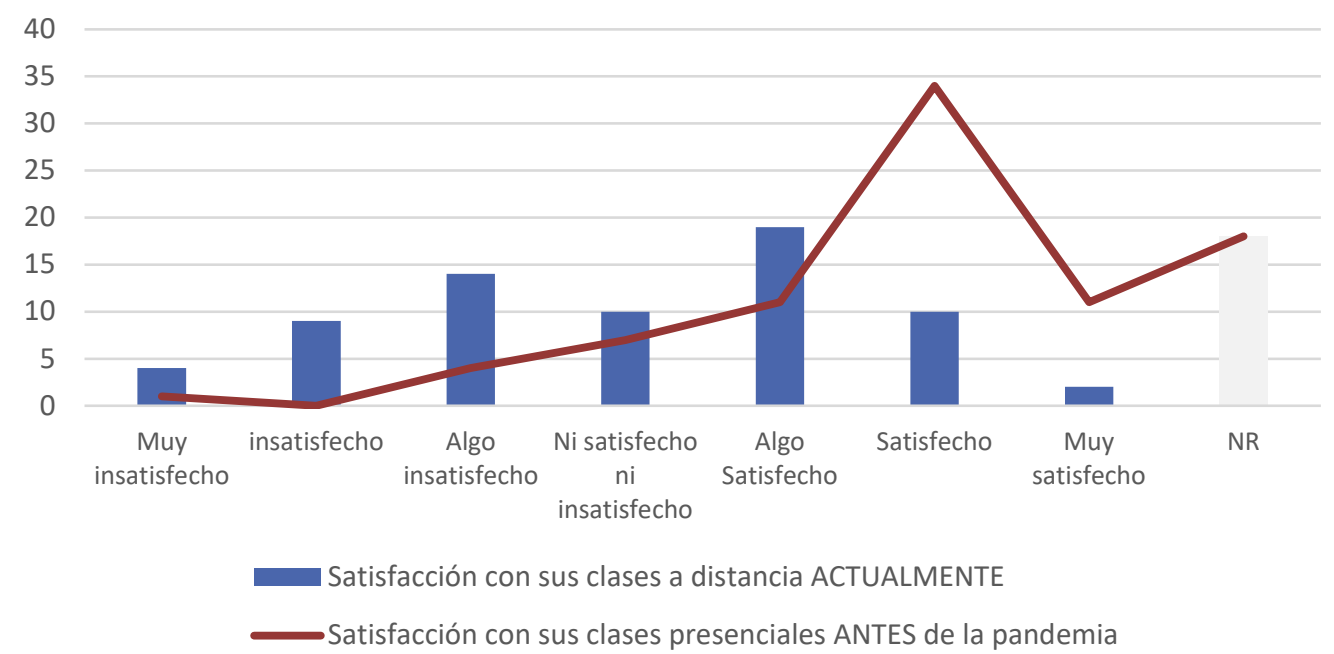

Fig. 4 Satisfacción con las clases presenciales (antes de la pandemia) y a distancia.

En general, las clases a distancia se perciben de menor calidad entre ambos grupos de estudiantes. Debido a aspectos como la falta de comunicación con los profesores, quienes deben esforzarse más y están menos motivados, además de la falta de prácticas.

La valoración general de otros aspectos como: la sobrecarga académica para los alumnos en las clases virtuales, la adecuación de los exámenes, la retroalimentación de las tareas, la poca flexibilidad en los plazos de entrega, y la calidad de las tareas y exámenes, es inferior en las clases a distancia (Anexo 1).

La interacción con otros estudiantes es un aspecto que también preocupa al alumnado, quienes consideran que, dada la menor interacción en una clase virtual, la interactividad entre compañeros y con el profesor es muy poca, es muy difícil realizar tareas grupales, no hay espacios para intercambiar ideas con los compañeros de clase, y no es posible formar grupos de estudio (Anexo 2).

\subsection{Conectividad e infraestructura}

En cuanto a la infraestructura requerida para el desarrollo de la docencia online, los participantes de ambas universidades manifestaron que cuentan con conexión a internet en su vivienda. La mayoría con la velocidad adecuada para las clases (56\%). Aunque un $24 \%$ tiene problemas con la velocidad de su internet, y el $5 \%$ no tienen acceso a un ordenador.

El alumnado de la UPV percibe menos problemas técnicos durante el acceso a clases y/o al material de estudio. Aunque desde ambas instituciones se valora positivamente la infraestructura de conexión para la enseñanza virtual (Figs. 5 y 6 ). 


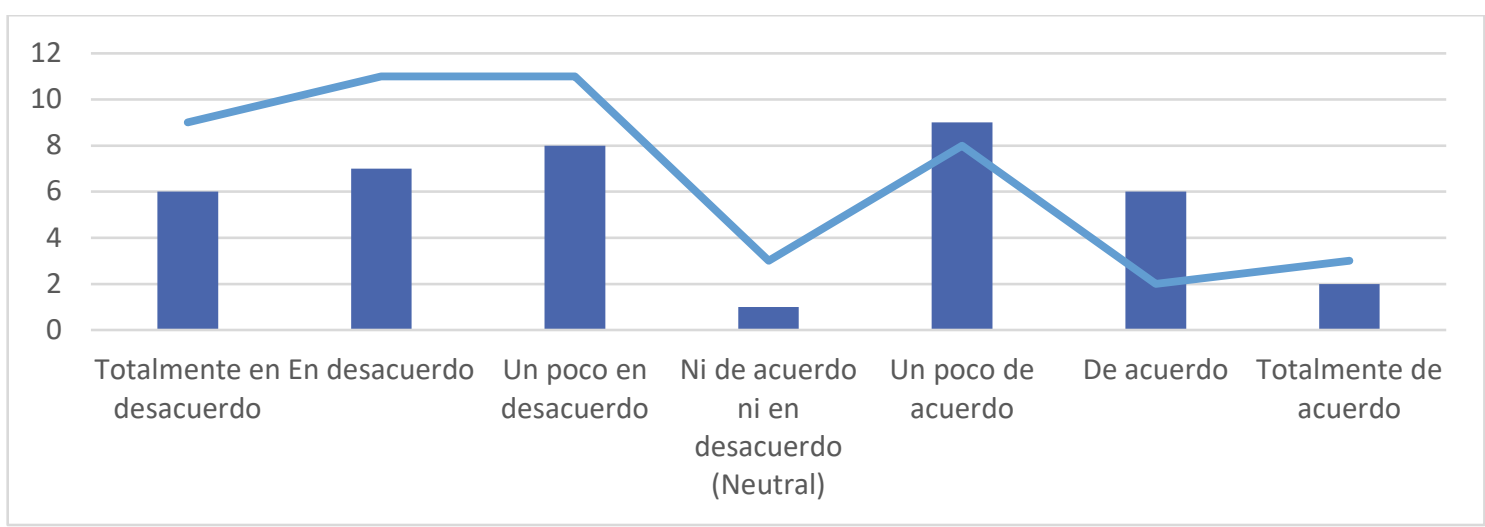

Fig. 5 Muchos problemas técnicos durante el acceso a clases o material de estudio

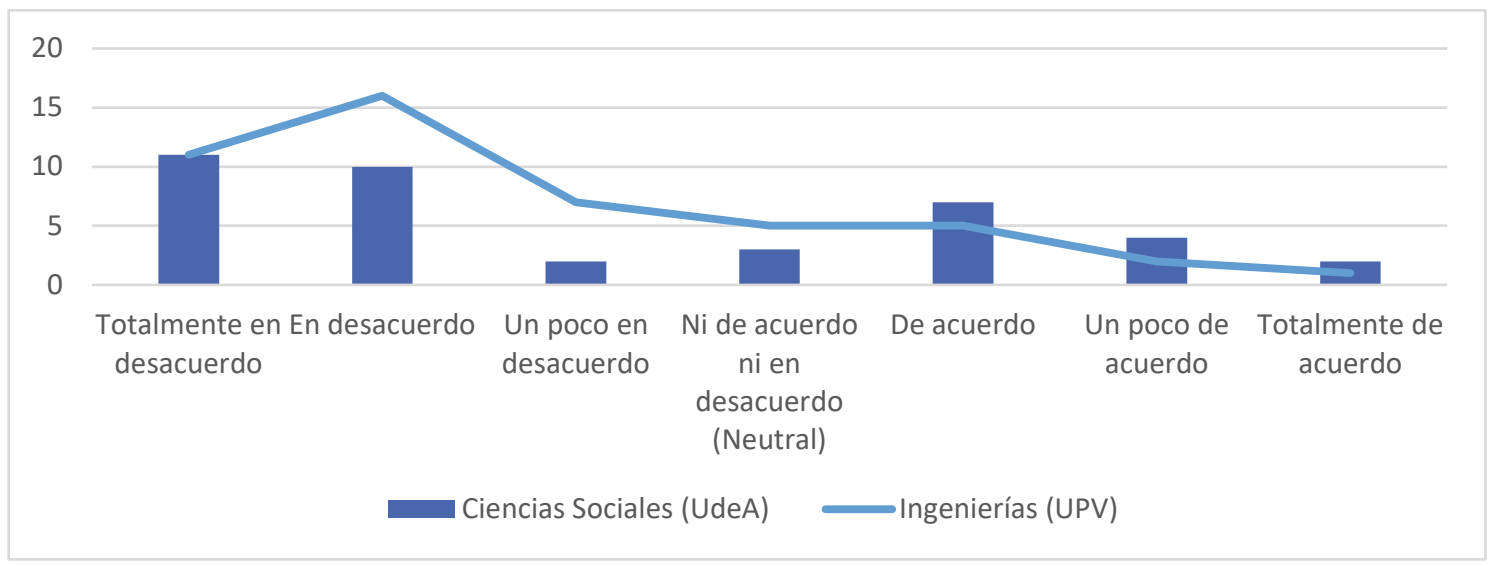

Fig. 6 Mi institución educativa no tiene la infraestructura de conexión (p. ej. servidores) adecuada para la enseñanza virtual

Respecto a la plataforma educativa utilizada, el $68 \%$ de quienes participaron consideran que es adecuada mientras que aproximadamente el $15 \%$ considera que no lo es.

Con relación al manejo de la plataforma por parte de los profesores, la opinión se divide, $45 \%$ del alumnado considera que los profesores no saben usar la plataforma educativa utilizada, mientras que el $42 \%$ considera que si. La opinión del alumnado de la UPV se inclina más a favor de las y los profesores. Mientras que la opinión entre los estudiantes de ciencias sociales señala deficiencias en el manejo de la plataforma educativa.

Respecto a la información disponible para el uso de la plataforma educativa, los participantes de la UPV valoran positivamente la disponibilidad de la información.

El 44\% del alumnado participante en el estudio considera que la falta de acceso a los libros de la biblioteca constituye una seria limitación; así mismo, el $67 \%$ cree que la falta de acceso a los laboratorios junto a la falta de acceso a más material de estudio (p. ej. PPTs) además de las grabaciones de la clase, representan algunas de las principales problemáticas. Finalmente, desde la UPV se valora mejor el acceso a recursos de enseñanza en la docencia virtual, mientras que en la UdeA los estudiantes consideran que es menor. 


\subsection{Entorno y ambiente de estudio}

En relación con el entorno de estudio y el espacio físico disponible en sus viviendas para las clases, el 78\% lo considera adecuado. Sin embargo, el $49 \%$ cree que las actividades de las demás personas en sus viviendas producen mucho ruido e interrupciones. Para el 79\% el mobiliario no es adecuado (p. ej. escritorio, silla), aunque el $50 \%$ considera que la infraestructura física de la que disponen en sus viviendas para las clases virtuales es mejor que en la institución educativa (Anexo 3).

\subsection{Problemas personales}

Con respecto a la disposición para las clases, el alumnado está de acuerdo en que es difícil concentrarse porque hay muchas distracciones ("móviles, ruidos e interrupciones en mi vivienda"), las largas jornadas frente al ordenador producen dolor de espalda, cansancio visual y físico en general, lo que hace que se sea difícil aceptar el nuevo contexto virtual y por lo tanto motivarse por esta modalidad a distancia (Anexo 4).

Respecto a la vida personal y la conciliación, para la mayoría no es fácil balancear las clases virtuales con las tareas/vida doméstica, aunque si es posible, pero difícil para algunos, tener un hobby o practicar un deporte. La percepción general es que las clases a distancia no dan espacios de recreación (60\%) por lo que, en general, causan más estrés que las presenciales (49\%) (Anexo 5).

En referencia al estado de ánimo, al menos el $69 \%$ del alumnado se ha visto directamente afectados por la pandemia, al haber enfermado del COVID-19 directamente ellos, algún amigo y/o familiar cercano. Esto denota algunos de los desafíos que a nivel personal los y las estudiantes han tenido que enfrentar durante la emergencia sanitaria, afectando también su desempeño académico y el interés por las clases.

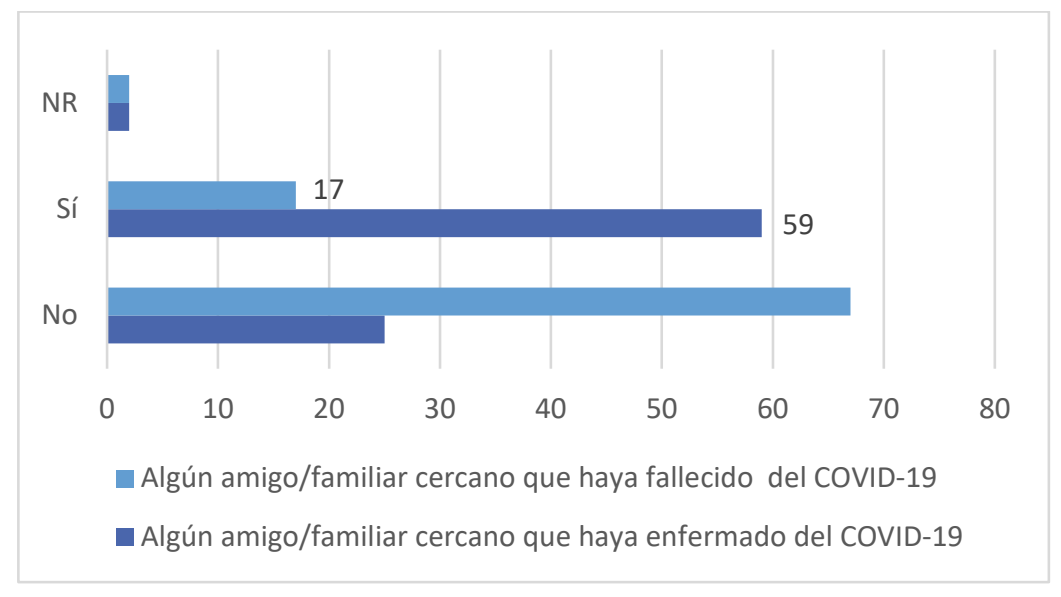

Fig. 7 Familiares/amigos afectados por el COVID-19

Para la mayoría la cuarentena es deprimente $(70 \%)$, la situación de incertidumbre de salud les afecta bastante (60\%). Algunos han manifestado estar o haber estado preocupados por familiares/amigos que han enfermado (50\%), o tristes por familiares/amigos que han fallecido (38\%), o intranquilos por no haber podido llevar a cabo sus revisiones/tratamientos médicos (33\%).

Finalmente, también se percibe un desinterés por parte de las instituciones educativas por la salud emocional de sus estudiantes (62\%) (Anexo 6). 


\section{Conclusiones}

El desarrollo del estudio ha permitido responder ampliamente a la pregunta planteada con relación a la percepción del alumnado matriculado en estudios de educación superior en España sobre los efectos de la pandemia en la docencia recibida y su rendimiento.

las distintas percepciones del alumnado se exploraron a través de cuatro dimensiones. En general los desafíos y problemáticas a los que se enfrentan soy muy similares entre el grupo de participante. La mayor diferencia se encontró en la percepción de algunos recursos disponibles por cada universidad, sin embargo, la percepción general de aspectos como calidad, motivación y desafíos personales son muy parecidos entre los grupos consultados (mujeres y hombres en ciencias sociales e ingenierías). Por otra parte, aunque la composición de la población de estudio refleja el sesgo de género propio de las ciencias sociales y la ingeniería, no se encontraron diferencias de género significativas respecto a los efectos de la pandemia sobre el alumnado universitario.

Desafortunadamente una discusión más amplia de los resultados se extiende más allá de las limitaciones de extensión de este trabajo, sin embargo, la reflexión y las discusiones generadas a partir de los resultados, nos ha permitido esbozar algunas lecciones aprendidas sobre la educación en la pandemia que resaltaremos a continuación, y que esperamos sirvan para la mejora de este proceso en un horizonte temporal más amplio.

Estas lecciones van encaminadas a solventar el principal problema manifestado por el alumnado sobre la falta de motivación y de concentración para seguir de una manera exitosa el desarrollo de las clases, al igual que se hacía en la docencia presencial. Las principales lecciones que recogemos son:

- Es necesario comprender las capacidades y competencias diferentes que a nivel digital puede tener el alumnado y los profesores, especialmente relacionado con el acceso digital.

- Desarrollo de prácticas diversas que potencien la participación en clase. Supone realizar un esfuerzo mayor que en la docencia presencial, pero es fundamental para un correcto desarrollo de las sesiones.

- El alumnado puede no aprender al mismo ritmo que cuando se trata de clases presenciales. Es necesario tenerlo en consideración e incidir en ello para generar un clima propicio para realizar preguntas y dudas.

- Realizar un entorno virtual lo más atractivo posible que fomente la participación.

- Promover un mayor seguimiento a la salud mental y el estado de ánimo de estudiantes y profesores.

Como líneas de investigación a desarrollar a partir de este trabajo, vale la pena mencionar, que este estudio se está llevando a cabo en otras instituciones de Perú y Estado Unidos, lo que permitirá realizar una comparativa interesante entre diferentes contextos. Así mismo, los aprendizajes descritos en este trabajo pueden servir de input para diseñar estrategias que enriquezcan el modelo de docencia en el contexto de la pandemia. Otra línea por trabajar a partir de este estudio abordaría la identificación de los canales que permitan la incorporación de estos aprendizajes en el modelo educativo. Finalmente, los resultados, conclusiones y las recomendaciones descritas en este estudio se compartirán con los docentes y alumnos, con el propósito de continuar la reflexión y posiblemente que se apliquen acciones de mejora. 


\section{Referencias}

Area Moreira, M., Bethencourt Aguilar, A., \& Martín Gómez, S. (2020). De la enseñanza semipresencial a la enseñanza online en tiempos de Covid-19: visiones del alumnado. Campus Virtuales: Revista Cientifica Iberoamericana de Tecnología Educativa, 9, 35-50.

Bertea, P. (2009). Measuring Students' Attitude Towards E-Learning . a Case Study. The 5th International Scientific Conference ELearning and Software for Education, 8.

Campbell, D., Picard-Aitken, M., Cote, G., Caruso, J., Valentim, R., Edmonds, S., ... Archambault, E. (2010). Bibliometrics as a Performance Measurement Tool for Research Evaluation: The Case of Research Funded by the National Cancer Institute of Canada. AMERICAN JOURNAL OF EVALUATION, 31(1), 66-83. https://doi.org/10.1177/1098214009354774

Commission, E. (2011). El género en la investigación. https://doi.org/10.2777/23655

Concha Vergara, M. H. (2014). E-Learning . The Educational Revolution. Revista Venezolana de Información, Tecnología $\quad y \quad$ Conocimiento, 11(2), $115-125 . \quad$ Retrieved from https://www.google.com/url?sa=t\&rct=j\&q=\&esrc=s\&source=web\&cd=\&ved=2ahUKEwj759Lp0orwAhVClJ UCHbK_ARkQFjAJegQIAhAD\&url=https\%3A\%2F\%2Fdialnet.unirioja.es\%2Fdescarga $\% 2$ Farticulo\%2F510 1940.pdf\&usg=AOvVaw0IzhwTquEeoqBzWiMbEUo8

Dixon, R., Dixon, K. C., \& Siragusa, L. (2007). Individuals' perceptions of online environments: What adult learners are telling us. ASCILITE 2007 - The Australasian Society for Computers in Learning in Tertiary Education, (2002), 207-218.

Durán-Rodríguez, R.-A. (2015). La Educación Virtual Universitaria como medio para mejorar las competencias. Universidad Politécnica de Cataluña.

European Commission. (2019). She Figures 2018. Report. https://doi.org/10.2777/936

González, M., Marco, E., \& Medina, T. (2020). Informe de iniciativas y herramientas de evaluación online universitaria en el contexto del Covid-19.

Ismaili, Y. (2021). Evaluation of students' attitude toward distance learning during the pandemic (Covid-19): a case study of ELTE university. On the Horizon, ahead-of-print(ahead-of-print). https://doi.org/10.1108/OTH09-2020-0032

Molas, J., \& Castro-Martínez, E. (2007). Ambiguity and conflict in the development of "Third Mission" indicators. Research Evaluation, 16. https://doi.org/10.3152/095820207X263592

Mu, E. (2020). What if Classroom Instruction Becomes a Thing of the Past? International Journal of the Analytic Hierarchy Process, (Vol. 12 No. 2 (2020)). Retrieved from https://doi.org/10.13033/ijahp.v12i2.805

Scull, J., Phillips, M., Sharma, U., \& Garnier, K. (2020). Innovations in teacher education at the time of COVID19: an Australian perspective. Journal of Education for Teaching, 46(4), 497-506. https://doi.org/10.1080/02607476.2020.1802701

Stoet, G., \& Geary, D. C. (2018). The Gender-Equality Paradox in Science, Technology, Engineering, and Mathematics Education. Psychological Science, 29(4), 581-593. https://doi.org/10.1177/0956797617741719

Struyven, K., Dochy, F., \& Janssens, S. (2005). Students' perceptions about evaluation and assessment in higher education: a review. Assessment \& Evaluation in Higher Education, 30(4), $325-341$. https://doi.org/10.1080/02602930500099102 


\section{Anexos}

Anexo 1. Hay una sobrecarga académica para los
alumnos en las clases virtuales

Los exámenes no son adecundos para

No hay una buena retroalimentación de

No hay flexibilidad de los profesores en

La calidad de las tareas y exámenes es clases online las tareas inferior en las clases virtuales

Totalmente en desacuerdo
En desacuerdo
Un poco en desacuerdo
Ni de acuerdo ni en desacuerdo (Neutral)
Un poco de acuerdo
De acuerdo
Totalmente de acuerdo
NR

\begin{tabular}{|c|c|c|c|c|c|c|c|c|c|c|c|c|c|c|c|}
\hline $\begin{array}{l}\text { Ciencias } \\
\text { Sociales } \\
\text { (UdeA) }\end{array}$ & $\begin{array}{l}\text { Ingenierias } \\
\text { (UPV) }\end{array}$ & Total & $\begin{array}{l}\text { Ciencias } \\
\text { Sociales } \\
\text { (UdeA) }\end{array}$ & $\begin{array}{l}\text { Ingenierias } \\
\text { (UPV) }\end{array}$ & Total & $\begin{array}{l}\text { Ciencias } \\
\text { Sociales } \\
\text { (UdeA) }\end{array}$ & $\begin{array}{l}\text { Ingenierias } \\
\text { (UPV) }\end{array}$ & Total & $\begin{array}{l}\text { Ciencias } \\
\text { Sociales } \\
\text { (UdeA) }\end{array}$ & $\begin{array}{l}\text { Ingenierias } \\
\text { (UPV) }\end{array}$ & Total & $\begin{array}{l}\text { Ciencias } \\
\text { Sociales } \\
\text { (UdeA) }\end{array}$ & $\begin{array}{l}\text { Ingenierias } \\
\text { (UPV) }\end{array}$ & Total & \\
\hline $5,1 \%$ & $6,4 \%$ & $5,8 \%$ & $10,3 \%$ & $4,3 \%$ & $7,0 \%$ & $2,6 \%$ & $4,3 \%$ & $3,5 \%$ & $15,4 \%$ & $4,3 \%$ & $9,3 \%$ & $10,3 \%$ & $12,8 \%$ & & $11,6 \%$ \\
\hline $7,7 \%$ & $4,3 \%$ & $5,8 \%$ & $10,3 \%$ & $8,5 \%$ & $9,3 \%$ & $10,3 \%$ & $4,3 \%$ & $7,0 \%$ & $10,3 \%$ & $6,4 \%$ & $8,1 \%$ & $12,8 \%$ & $10,6 \%$ & & $11,6 \%$ \\
\hline $10,3 \%$ & $4,3 \%$ & $7,0 \%$ & $5,1 \% \mid$ & $2,1 \% \mid$ & $3,5 \%$ & $2,6 \%$ & $6,4 \%$ & $4,7 \%$ & $12,8 \%$ & $12,8 \%$ & $12,8 \%$ & $5,1 \%$ & $4,3 \%$ & & 4,7 \\
\hline $5,1 \%$ & $14,9 \%$ & $10,5 \%$ & $10,3 \%$ & $14,9 \%$ & $12,8 \%$ & $20,5 \%$ & $12,8 \%$ & $16,3 \%$ & $10,3 \%$ & $12,8 \%$ & $11,6 \%$ & $15,4 \%$ & $14,9 \%$ & & $15,1 \%$ \\
\hline $15,4 \%$ & $17,0 \%$ & $16,3 \%$ & $17,9 \%$ & $19,1 \%$ & $18,6 \%$ & $17,9 \%$ & $23,4 \%$ & $20,9 \%$ & $15,4 \%$ & $25,5 \%$ & $20,9 \%$ & $12,8 \%$ & $21,3 \%$ & & $17,4 \%$ \\
\hline $17,9 \%$ & $19,1 \%$ & $18,6 \%$ & $12,8 \%$ & $14,9 \%$ & $14,0 \%$ & $20,5 \%$ & $19,1 \%$ & $19,8 \%$ & $17,9 \%$ & $8,5 \%$ & $12,8 \%$ & $20,5 \%$ & $12,8 \%$ & & $16,3^{\circ}$ \\
\hline $33,3 \%$ & $25,5 \%$ & $29,1 \%$ & $28,2 \%$ & $27,7 \%$ & $27,9 \%$ & $20,5 \%$ & $21,3 \%$ & $20,9 \%$ & $12,8 \%$ & $21,3 \%$ & $17,4 \%$ & $17,9 \%$ & $14,9 \%$ & & $16,3 \%$ \\
\hline $5,1 \%$ & $8,5 \%$ & $7,0 \%$ & $5,1 \%$ & $8,5 \%$ & $7,0 \%$ & $5,1 \%$ & $8,5 \%$ & $7,0 \%$ & $5,1 \%$ & $8,5 \%$ & $7,0 \%$ & $5,1 \%$ & $8,5 \%$ & & 7, \\
\hline
\end{tabular}

Ciencias

\begin{tabular}{l|l|l|l|} 
Cociales & Ciencias \\
Sociales & Ingenierias & Socias
\end{tabular}

Anexo 2.

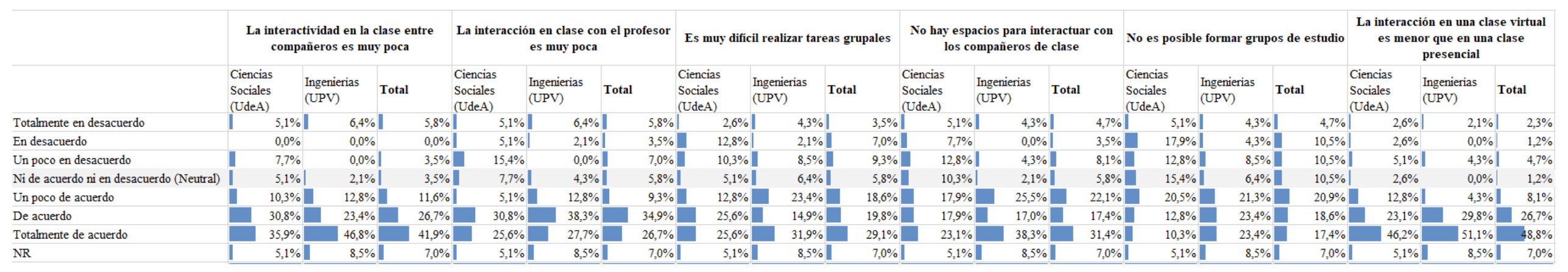

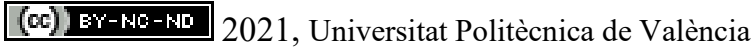


Anexo 3.

\begin{tabular}{|c|c|c|c|c|c|c|c|c|c|c|c|c|}
\hline & \multicolumn{3}{|c|}{$\begin{array}{l}\text { Mi vivienda no tiene espacio físico } \\
\text { adecuado para mis clases virtuales }\end{array}$} & \multicolumn{3}{|c|}{$\begin{array}{l}\text { Las actividades de las demás personas } \\
\text { en mi vivienda producen mucho ruido e } \\
\text { interrupciones }\end{array}$} & \multicolumn{3}{|c|}{$\begin{array}{l}\text { No tengo el mobiliario adecuado en mi } \\
\text { vivienda (p. ej. escritorio, silla) para mis } \\
\text { clases virtuales }\end{array}$} & \multicolumn{3}{|c|}{$\begin{array}{c}\text { La infraestructura fisica de la que } \\
\text { dispongo en mi vivienda para las clases } \\
\text { virtuales es peor que en la institución } \\
\text { educativa }\end{array}$} \\
\hline & $\begin{array}{l}\text { Ciencias } \\
\text { Sociales } \\
\text { (UdeA) }\end{array}$ & $\begin{array}{l}\text { Ingenierias } \\
\text { (UPV) }\end{array}$ & Total & $\begin{array}{l}\text { Ciencias } \\
\text { Sociales } \\
\text { (UdeA) }\end{array}$ & $\begin{array}{l}\text { Ingenierias } \\
\text { (UPV) }\end{array}$ & Total & $\begin{array}{l}\text { Ciencias } \\
\text { Sociales } \\
\text { (UdeA) }\end{array}$ & $\begin{array}{l}\text { Ingenierias } \\
\text { (UPV) }\end{array}$ & Total & $\begin{array}{l}\text { Ciencias } \\
\text { Sociales } \\
\text { (UdeA) }\end{array}$ & $\begin{array}{l}\text { Ingenierias } \\
\text { (UPV) }\end{array}$ & Total \\
\hline Totalmente en desacuerdo & $43,6 \%$ & $44,7 \%$ & $44,2 \%$ & $15,4 \%$ & $23,4 \%$ & $19,8 \%$ & $51,3 \%$ & $44,7 \%$ & $47,7 \%$ & $25,6 \%$ & $29,8 \%$ & $27,9 \%$ \\
\hline En desacuerdo & $20,5 \%$ & $27,7 \%$ & $24,4 \%$ & $17,9 \%$ & $14,9 \%$ & $16,3 \%$ & $20,5 \%$ & $23,4 \%$ & $22,1 \%$ & $23,1 \%$ & $8,5 \%$ & $15,1 \%$ \\
\hline Un poco en desacuerdo & $10,3 \%$ & $8,5 \%$ & $9,3 \%$ & $7,7 \%$ & $6,4 \%$ & $7,0 \%$ & $2,6 \%$ & $14,9 \%$ & $9,3 \%$ & $12,8 \%$ & $2,1 \%$ & $7,0 \%$ \\
\hline Ni de acuerdo ni en desacuerdo (Neutral) & $0,0 \%$ & $6,4 \%$ & $3,5 \%$ & $7,7 \%$ & $4,3 \%$ & $5,8 \%$ & $7,7 \%$ & $4,3 \%$ & $5,8 \%$ & $10,3 \%$ & $17,0 \%$ & $14,0 \%$ \\
\hline Un poco de acuerdo & $5,1 \%$ & $6,4 \%$ & $5,8 \%$ & $23,1 \%$ & $25,5 \%$ & $24,4 \%$ & $7,7 \%$ & $8,5 \%$ & $8,1 \%$ & $7,7 \%$ & $10,6 \%$ & $9,3 \%$ \\
\hline De acuerdo & $7,7 \%$ & $4,3 \%$ & $5,8 \%$ & $10,3 \%$ & $8,5 \%$ & $9,3 \%$ & $5,1 \%$ & $2,1 \%$ & $3,5 \%$ & $10,3 \%$ & $19,1 \%$ & $15,1 \%$ \\
\hline Totalmente de acuerdo & $7,7 \%$ & $2,1 \%$ & $4,7 \%$ & $12,8 \%$ & $17,0 \%$ & $15,1 \%$ & $0,0 \%$ & $2,1 \%$ & $1,2 \%$ & $5,1 \%$ & $12,8 \%$ & $9,3 \%$ \\
\hline NR & $5,1 \%$ & $0,0 \%$ & $2,3 \%$ & $5,1 \%$ & $0,0 \%$ & $2,3 \%$ & $5,1 \%$ & $0,0 \%$ & $2,3 \%$ & $5,1 \%$ & $0,0 \%$ & $2,3 \%$ \\
\hline
\end{tabular}

Anexo 4.

\begin{tabular}{|c|c|c|c|c|c|c|c|c|c|c|c|c|}
\hline & \multicolumn{3}{|c|}{$\begin{array}{c}\text { Es difícil concentrarse porque hay } \\
\text { muchas distracciones (móviles, ruidos e } \\
\text { interrupciones en mi vivienda) }\end{array}$} & \multicolumn{3}{|c|}{$\begin{array}{c}\text { Largas horas frente al ordenador producen } \\
\text { dolor de espalda, cans ancio visual y físico } \\
\text { en general }\end{array}$} & \multicolumn{3}{|c|}{ Es difícil aceptar el nuevo contexto virtual } & \multicolumn{3}{|c|}{$\begin{array}{l}\text { No es fácil mantenerse motivados en las } \\
\text { clases virtuales }\end{array}$} \\
\hline & $\begin{array}{l}\text { Ciencias } \\
\text { Sociales } \\
\text { (UdeA) }\end{array}$ & $\begin{array}{l}\text { Ingenierías } \\
\text { (UPV) }\end{array}$ & Total & $\begin{array}{l}\text { Ciencias } \\
\text { Sociales } \\
\text { (UdeA) }\end{array}$ & $\begin{array}{l}\text { Ingenierías } \\
\text { (UPV) }\end{array}$ & Total & $\begin{array}{l}\text { Ciencias } \\
\text { Sociales } \\
\text { (UdeA) }\end{array}$ & $\begin{array}{l}\text { Ingenierías } \\
\text { (UPV) }\end{array}$ & Total & $\begin{array}{l}\text { Ciencias } \\
\text { Sociales } \\
\text { (UdeA) }\end{array}$ & $\begin{array}{l}\text { Ingenierías } \\
\text { (UPV) }\end{array}$ & Total \\
\hline Totalmente en desacuerdo & $5,1 \%$ & $8,5 \%$ & $7,0 \%$ & $0,0 \%$ & $2,1 \%$ & $1,2 \%$ & $7,7 \%$ & $2,1 \%$ & $4,7 \%$ & $0,0 \%$ & $2,1 \%$ & $1,2 \%$ \\
\hline En desacuerdo & $5,1 \%$ & $2,1 \%$ & $3,5 \%$ & $2,6 \%$ & $4,3 \%$ & $3,5 \%$ & $2,6 \%$ & $6,4 \%$ & $4,7 \%$ & $7,7 \%$ & $2,1 \%$ & $4,7 \%$ \\
\hline Un poco en desacuerdo & $2,6 \%$ & $2,1 \%$ & $2,3 \%$ & $2,6 \%$ & $2,1 \%$ & $2,3 \%$ & $10,3 \%$ & $6,4 \%$ & $8,1 \%$ & $2,6 \%$ & $2,1 \%$ & $2,3 \%$ \\
\hline $\begin{array}{l}\text { Ni de acuerdo ni en desacuerdo } \\
\text { (Neutral) }\end{array}$ & $0,0 \%$ & $6,4 \%$ & $3,5 \%$ & $0,0 \%$ & $4,3 \%$ & $2,3 \%$ & $10,3 \%$ & $14,9 \%$ & $12,8 \%$ & $2,6 \%$ & $6,4 \%$ & $4,7 \%$ \\
\hline Un poco de acuerdo & $15,4 \%$ & $10,6 \%$ & $12,8 \%$ & $17,9 \%$ & $12,8 \%$ & $15,1 \%$ & $12,8 \%$ & $17,0 \%$ & $15,1 \%$ & $12,8 \%$ & $17,0 \%$ & $15,1 \%$ \\
\hline De acuerdo & $23,1 \%$ & $21,3 \%$ & $22,1 \%$ & $12,8 \%$ & $14,9 \%$ & $14,0 \%$ & $17,9 \%$ & $14,9 \%$ & $16,3 \%$ & $15,4 \%$ & $8,5 \%$ & $11,6 \%$ \\
\hline Totalmente de acuerdo & $30,8 \%$ & $27,7 \%$ & $29,1 \%$ & $46,2 \%$ & $38,3 \%$ & $41,9 \%$ & $20,5 \%$ & $17,0 \%$ & $18,6 \%$ & $41,0 \%$ & $40,4 \%$ & $40,7 \%$ \\
\hline NR & $17,9 \%$ & $21,3 \%$ & $19,8 \%$ & $17,9 \%$ & $21,3 \%$ & $19,8 \%$ & $17,9 \%$ & $21,3 \%$ & $19,8 \%$ & $17,9 \%$ & $21,3 \%$ & $19,8 \%$ \\
\hline
\end{tabular}

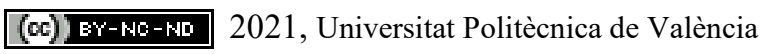


Anexo 5.

\begin{tabular}{|c|c|c|c|c|c|c|c|c|c|c|c|c|}
\hline & \multicolumn{3}{|c|}{$\begin{array}{l}\text { No es fácil balancear las clases virtuales } \\
\text { con las tareas/vida domés tica }\end{array}$} & \multicolumn{3}{|c|}{$\begin{array}{l}\text { No es fácil tener un hobby o practicar un } \\
\text { deporte con las clases virtuales }\end{array}$} & \multicolumn{3}{|c|}{$\begin{array}{l}\text { Tengo que conciliar mis estudios } \\
\text { virtuales on el cuidado de niños } \\
\text { menores o adultos mayores }\end{array}$} & \multicolumn{3}{|c|}{$\begin{array}{c}\text { Es más difícil organizarse cuando } \\
\text { se llevan clases virtuales }\end{array}$} \\
\hline & $\begin{array}{l}\text { Ciencias } \\
\text { Sociales } \\
\text { (UdeA) }\end{array}$ & $\begin{array}{l}\text { Ingenierías } \\
\text { (UPV) }\end{array}$ & Total & $\begin{array}{l}\text { Ciencias } \\
\text { Sociales } \\
\text { (UdeA) }\end{array}$ & $\begin{array}{l}\text { Ingenierías } \\
\text { (UPV) }\end{array}$ & Total & $\begin{array}{l}\text { Ciencias } \\
\text { Sociales } \\
(\text { UdeA) }\end{array}$ & $\begin{array}{l}\text { Ingenierías } \\
\text { (UPV) }\end{array}$ & Total & $\begin{array}{l}\text { Ciencias } \\
\text { Sociales } \\
\text { (UdeA) }\end{array}$ & $\begin{array}{l}\text { Ingenierías } \\
\text { (UPV) }\end{array}$ & Total \\
\hline Totalmente en desacuerdo & $10,3 \%$ & $2,1 \%$ & $5,8 \%$ & $17,9 \%$ & $8,5 \%$ & $12,8 \%$ & $46,2 \%$ & $42,6 \%$ & $44,2 \%$ & $23,1 \%$ & $8,5 \%$ & $15,1 \%$ \\
\hline En desacuerdo & $7,7 \%$ & $12,8 \%$ & $10,5 \%$ & $20,5 \%$ & $12,8 \%$ & $16,3 \%$ & $10,3 \%$ & $8,5 \%$ & $9,3 \%$ & $12,8 \%$ & $19,1 \%$ & $16,3 \%$ \\
\hline Un poco en desacuerdo & $12,8 \%$ & $8,5 \%$ & $10,5 \%$ & $7,7 \%$ & $10,6 \%$ & $9,3 \%$ & $0,0 \%$ & $0,0 \%$ & $0,0 \%$ & $10,3 \%$ & $14,9 \%$ & $12,8 \%$ \\
\hline $\begin{array}{l}\text { Ni de acuerdo ni en desacuerdo } \\
\text { (Neutral) }\end{array}$ & $5,1 \%$ & $4,3 \%$ & $4,7 \%$ & $10,3 \%$ & $4,3 \%$ & $7,0 \%$ & $10,3 \%$ & $8,5 \%$ & $9,3 \%$ & $15,4 \%$ & $6,4 \%$ & $10,5 \%$ \\
\hline Un poco de acuerdo & $10,3 \%$ & $17,0 \%$ & $14,0 \%$ & $7,7 \%$ & $21,3 \%$ & $15,1 \%$ & $7,7 \%$ & $12,8 \%$ & $10,5 \%$ & $5,1 \%$ & $10,6 \%$ & $8,1 \%$ \\
\hline De acuerdo & $17,9 \%$ & $21,3 \%$ & $19,8 \%$ & $7,7 \%$ & $17,0 \%$ & $12,8 \%$ & $7,7 \%$ & $4,3 \%$ & $5,8 \%$ & $15,4 \%$ & $6,4 \%$ & $10,5 \%$ \\
\hline Totalmente de acuerdo & $23,1 \%$ & $12,8 \%$ & $17,4 \%$ & $15,4 \%$ & $4,3 \%$ & $9,3 \%$ & $5,1 \%$ & $2,1 \%$ & $3,5 \%$ & $5,1 \%$ & $12,8 \%$ & $9,3 \%$ \\
\hline NR & $12,8 \%$ & $21,3 \%$ & $17,4 \%$ & $12,8 \%$ & $21,3 \%$ & $17,4 \%$ & $12,8 \%$ & $21,3 \%$ & $17,4 \%$ & $12,8 \%$ & $21,3 \%$ & $17,4 \%$ \\
\hline
\end{tabular}

\section{Anexo 6.}

\begin{tabular}{|c|c|c|c|c|c|c|c|c|c|c|c|c|c|c|c|c|c|c|c|c|c|c|c|c|c|}
\hline & \multicolumn{3}{|c|}{ La cuarentena es deprimente } & \multicolumn{3}{|c|}{$\begin{array}{l}\text { La situación de incertidumbre de } \\
\text { salud me afecta bastante }\end{array}$} & \multicolumn{3}{|c|}{$\begin{array}{l}\text { No he podido llevar a cabo mis } \\
\text { revisiones/tratamientos médicos }\end{array}$} & \multicolumn{3}{|c|}{$\begin{array}{l}\text { Estoy preocupado por } \\
\text { familiares/amigos que han } \\
\text { enfermado }\end{array}$} & \multicolumn{3}{|c|}{$\begin{array}{c}\text { Estoy triste por familiares/amigos } \\
\text { que han fallecido }\end{array}$} & \multicolumn{3}{|c|}{$\begin{array}{l}\text { A la escuela/universidad no les } \\
\text { importa la salud mental de los } \\
\text { estudiantes }\end{array}$} & \multicolumn{3}{|c|}{$\begin{array}{l}\text { Las clases virtuales no dan } \\
\text { espacios de recreación }\end{array}$} & \multicolumn{4}{|c|}{$\begin{array}{l}\text { Las clases virtuales, en general, } \\
\text { causan más estrés que las } \\
\text { presenciales }\end{array}$} \\
\hline & \begin{tabular}{l|l} 
Ciencias & In \\
Sociales & (1 \\
(UdeA) &
\end{tabular} & $\begin{array}{l}\text { Ingenierías } \\
\text { (UPV) }\end{array}$ & Total & \begin{tabular}{l|l} 
Ciencias & I \\
Sociales & ( \\
(UdeA) &
\end{tabular} & $\begin{array}{l}\text { Ingenierías } \\
\text { (UPV) }\end{array}$ & Total & $\begin{array}{l}\text { Ciencias } \\
\text { Sociales } \\
\text { (UdeA) }\end{array}$ & $\begin{array}{l}\text { Ingenierías } \\
\text { (UPV) }\end{array}$ & Total & \begin{tabular}{l|l} 
Ciencias & In \\
Sociales & (t \\
(UdeA) & (
\end{tabular} & $\begin{array}{l}\text { Ingenierías } \\
\text { (UPV) }\end{array}$ & Total & $\begin{array}{l}\text { Ciencias } \\
\text { Sociales } \\
\text { (UdeA) }\end{array}$ & $\begin{array}{l}\text { Ingenierías } \\
\text { (UPV) }\end{array}$ & Total & \begin{tabular}{l|l} 
Ciencias & In \\
Sociales & In \\
(UdeA) & (
\end{tabular} & $\begin{array}{l}\text { Ingenierías } \\
\text { (UPV) }\end{array}$ & is Total & \begin{tabular}{l|l} 
Ciencias & In \\
Sociales & (C \\
(UdeA) &
\end{tabular} & $\begin{array}{l}\text { Ingenierías } \\
\text { (UPV) }\end{array}$ & S Total & $\begin{array}{l}\text { Ciencias } \\
\text { Sociales } \\
\text { (UdeA) }\end{array}$ & & enierías $\mathrm{To}$ & Total \\
\hline Totalme & | $2,6 \%$ & $\quad 6,4 \%$ & $4,7 \%$ & $10,3 \%$ & $8,5 \%$ & $9,3 \%$ & $12,8 \%$ & $21,3 \%$ & $17,4 \%$ & $\quad 7,7 \%$ & $17,0 \%$ & $12,8 \%$ & $15,4 \%$ & $25,5 \%$ & $20,9 \%$ & $5,1 \%$ & $6,4 \%$ & $5,8 \%$ & $5,1 \%$ & $6,4 \%$ & $5,8 \%$ & $6 \quad 12,8 \%$ & & $6,4 \%$ & \\
\hline des & $7,7 \%$ & $0,0 \%$ 【 & $3,5 \%$ & $5,1 \%$ & $8,5 \%$ & $7,0 \%$ & $15,4 \%$ 【 & $4,3 \%$ & $9,3 \%$ 【 & $2,6 \%$ & $6,4 \%$ & $4,7 \%$ & $2,6 \% \|$ & $2,1 \% \|$ & $2,3 \%$ & $5,1 \%$ & $6,4 \%$ & $5,8 \%$ & $0,0 \%$ & $0,0 \%$ & $0,0 \%$ & $10,3 \%$ & & $12,8 \%$ & 11,6 \\
\hline Un poco & $0,0 \%$ | & $2,1 \%$ & $1,2 \%$ & $0,0 \%$ & $2,1 \%$ & $1,2 \%$ & $2,6 \%$ & $8,5 \%$ & $5,8 \%$ & $5,1 \%$ & $0,0 \% \|$ & $2,3 \%$ & $2,6 \%$ & $0,0 \%$ & $1,2 \%$ & $0,0 \%$ & $8,5 \%$ & $4,7 \%$ & $2,6 \%$ & $0,0 \%$ & $1,2 \%$ & $10,3 \%$ & & $0,0 \%$ & I \\
\hline & $2,6 \%$ & $2,1 \%$ & $2,3 \%$ & $2,6 \%$ & $8,5 \%$ & $5,8 \%$ & $12,8 \%$ & $19,1 \%$ & $16,3 \%$ & $12,8 \%$ & $12,8 \%$ & $12,8 \%$ & $20,5 \%$ & $19,1 \%$ & $19,8 \%$ & $7,7 \%$ & $2,1 \%$ & $4,7 \%$ & $17,9 \%$ & $12,8 \%$ & $15,1 \%$ & $5,1 \%$ & & $10,6 \%$ & 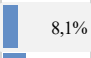 \\
\hline & $7,9 \%$ & 14,9 & $16,3 \%$ & $8 \%$ & 19, & & & & & & & & $7,7 \%$ & $4,3 \%$ & $\square$ & $15,4 \%$ & $23,4 \%$ & $19,8 \%$ & $12,8 \%$ & $17,0 \%$ & $15,1 \%$ & $7,7 \%$ & & $17,0 \%$ & . \\
\hline & 15,4 & 25,5 & $20,9 \%$ & $7,7 \%$ & & & & & & & & & 10 & & 12,8 & $25,6 \%$ & & & & & & & & $14,9 \%$ & 然, \\
\hline lotalmente de & $41,0 \%$ & $27,7 \%$ & $33,7 \%[$ & $48,7 \%$ & $23,4 \%$ & $34,9 \%$ & $12,8 \%$ & $8,5 \%$ & $10,5 \%$ & $33,3 \%$ & $1,0 \%$ & $24,4 \%[$ & $28,2 \%$ & $12,8 \%$ & $19,8 \%$ & $28,2 \%$ & $27,7 \%$ & $27,9 \%$ & $23,1 \%$ & $17,0 \%$ & $19,8 \%$ & $33,3 \%$ & & $17,0 \%$ & 4,4 \\
\hline $\mathrm{NR}$ & $12,8 \%$ & $21,3 \%$ & $17,4 \%$ & $12,8 \%$ & $21,3 \%$ & $17,4 \%$ & $12,8 \%$ & $21,3 \%$ & $17,4 \%$ & $12,8 \%$ & $21,3 \%$ & $17,4 \%$ & $12,8 \%$ & $21,3 \%$ & $17,4 \%$ & $12,8 \%$ & $21,3 \%$ & $\begin{array}{ll}6 \\
\end{array}$ & $12,8 \%$ & $21,3 \%$ & $17,4 \%$ & $12,8 \%$ & & $21,3 \%$ & 17,4 \\
\hline
\end{tabular}

(cc) EY-NC-ND 2021, Universitat Politècnica de València

Congreso In-Red (2021) 